\title{
REPRESENTATION THEOREM FOR THE SOLUTION OF WEAKLY HYPERBOLIC EQUATIONS WITH FAST OSCILLATING COEFFICIENTS
}

\author{
TAMOTU KINOSHITA \\ Institute of Mathematics, University of Tsukuba \\ 305-8571, Ibaraki, Japan \\ E-mail:kinosita@math.tsukuba.ac.jp \\ KAREN YAGDJIAN \\ Department of Mathematics, Kansas State University \\ 138 Cardwell Hall, Manhattan, KS 66506-2602, USA \\ E-mail: yagdjian@math.ksu.edu
}

Dedicated to Professor Sergio Spagnolo on the occasion of his 60th birthday

\begin{abstract}
This work is concerned with the influence of oscillations in weakly hyperbolic operators on the well-posedness of the Cauchy problem. The fundamental solution to the Cauchy problem is constructed for the equations with oscillations in the coefficient very close to the ones destroying the $C^{\infty}$ well-posedness.
\end{abstract}

1. Introduction. The subject of this paper concerns with the investigation of the influence of the oscillations in weakly hyperbolic operators on the well-posedness of the Cauchy problem. Since the example constructed by F. Colombini and S. Spagnolo [1], it is well-known that oscillations can break down the well-posedness. Namely, they constructed a second order equation $\partial_{t}^{2} u-a(t) \partial_{x}^{2} u=f(t, x)$, with the smooth coefficient $a \in C^{\infty}$, for which the Cauchy problem is not $C^{\infty}$ well-posed (see also [2]). The proof is based on the very delicate investigation of the energy of solutions. For the equation

$$
\partial_{t}^{2} u-\exp \left(-2 t^{-\alpha}\right) b\left(t^{-1}\right)^{2} \partial_{x}^{2} u=0, \quad \alpha=\text { const. }>0,
$$

where $b(s)$ is a non-constant, positive and smooth 1-periodic function on $\mathbb{R}$, the energy method convinces that the Cauchy problem is $C^{\infty}$ well-posed if $\alpha \geq 1$. S. Tarama [6]

2000 Mathematics Subject Classification: Primary 35C15, 35L15; Secondary 35L80.

The paper is in final form and no version of it will be published elsewhere. 
appeals to the Floquet theory to prove that the problem is $C^{\infty}$ well-posed if and only if $\alpha \geq 1 / 2$.

For second order equations with coefficients independent of the spatial variables some sufficient for the well-posedness conditions are given in [7]. The equation under consideration in [7] is the following:

$$
D_{t}^{2} u+\sum_{|\alpha|=2} a_{0, \alpha}(t) D_{x}^{\alpha} u+\sum_{j+|\alpha| \leq 1} a_{j, \alpha}(t) D_{t}^{j} D_{x}^{\alpha} u=f .
$$

It is supposed that the principal symbol can be written in the form

$$
\tau^{2}+\sum_{|\alpha|=2} a_{0, \alpha}(t) \xi^{\alpha}=\left(\tau-\lambda_{1}(t, \xi)\right)\left(\tau-\lambda_{2}(t, \xi)\right),
$$

with the real-valued functions $\lambda_{l}(t, \xi)(l=1,2)$ which satisfy the conditions

$$
\left|\lambda_{l}(t, \xi)\right| \leq c \lambda(t)|\xi|, \quad l=1,2, \quad\left|\lambda_{1}(t, \xi)-\lambda_{2}(t, \xi)\right| \geq \delta \lambda(t)|\xi|, \quad \delta=\text { const. }>0,
$$

for all $t \in[0, T], \xi \in \mathbb{R}_{\xi}^{n}$. Here $\lambda \in C^{2}([0, T]), \lambda(0)=\lambda^{\prime}(0)=0, \lambda^{\prime}(t)>0$ for $t>0$. Thus, at $t=0$ the operator has multiple characteristics. Furthermore, it is assumed in [7] that the following inequalities are satisfied:

$$
\begin{aligned}
& \left|D_{t}^{k} \operatorname{Re} a_{0, \alpha}(t)\right| \leq C \lambda^{2}(t)\left(\frac{|\log \lambda(t)|}{\Lambda(t)}\right)^{2-|\alpha|}\left(\frac{\lambda(t)|\log \lambda(t)|}{\Lambda(t)}\right)^{k}, \quad k=0,1,2, \\
& \left|D_{t}^{k} \operatorname{Im} a_{0, \alpha}(t)\right| \leq C \frac{\lambda^{2}(t)}{\Lambda(t)}\left(\frac{\lambda(t)|\log \lambda(t)|}{\Lambda(t)}\right)^{k}, \quad k=0,1,
\end{aligned}
$$

for all $t \in(0, T], 0<|\alpha| \leq 2$. Then in [7] it is proved that for equation (1.2) the Cauchy problem is $C^{\infty}$ well-posed. The conditions (1.4) and (1.5) couple together an oscillation with the degeneracy of the principal part.

For the equation (1.1) one can set $\lambda_{1}(t, \xi)=-\lambda_{2}(t, \xi)=\exp \left(-t^{-\alpha}\right) b\left(t^{-1}\right)|\xi|$, and $\lambda(t)=\exp \left(-t^{-\alpha}\right)$. Then the critical value $\alpha=1 / 2$ of (1.1) is reflected in (1.4) by the term $\left(\frac{\lambda(t)|\log \lambda(t)|}{\Lambda(t)}\right)^{k}$ containing $|\log \lambda(t)|$. Indeed, to satisfy that condition with $k=1$ we have to require $0<$ const. $\leq t^{2} \lambda(t)|\log \lambda(t)| / \Lambda(t)$ for all $t \in(0, T]$, which is equivalent to $\alpha \geq 1 / 2$. In [7] such equations are called equations with fast oscillating coefficients, while the equations with coefficients satisfying estimates with $\left(\frac{\lambda(t)}{\Lambda(t)}\right)^{k}$ (that corresponds to $\alpha \geq 1$ ) one can call possessing slowly oscillating coefficients. All other cases (corresponding to $\alpha<1 / 2$ ) can be regarded as very fast oscillating. Such classification is useful as well in completely other problem of $L_{p}-L_{q}$ decay (as $t \rightarrow \infty$ ) estimates for strictly hyperbolic equations (see [4]), where anew oscillations can have destructive consequences.

On the other hand after an investigation of the well-posedness the next interesting question is a construction of the fundamental solution (or of the parametrix) and a description of the propagation of singularities in the framework of the micro-local analysis. For the operators with slow oscillations such construction can be found in [8]. The goal of the present note is to fill up the gap for the equations with oscillations in the coefficients very close to the ones destroying the well-posedness. Thus in this paper we consider the critical case of equations with fast oscillating coefficients. 
Let $p=p(t)$ be a smooth function $p \in C^{\infty}(0, T](0<T<1)$, satisfying

$$
\begin{array}{ll}
p^{\prime}(t) \leq-\frac{\gamma}{t}\left({ }^{\exists} \gamma>1\right), & \forall t \in(0, T], \\
0 \leq p^{\prime \prime}(t) \leq{ }^{\exists} C\left|p(t) p^{\prime}(t)\right|^{2}, & \forall t \in(0, T] .
\end{array}
$$

Let $a(t)$ be a non-negative function such that

$$
a(t)=\lambda(t) b(t)
$$

where

$$
\lambda(t)= \begin{cases}e^{-p(t)} & \text { for } 0<t \leq T \\ 0 & \text { for } t=0\end{cases}
$$

belongs to $C^{1}[0, T]$ and $b(t)$ is a uniformly positive smooth function satisfying (1.6) $b \in C^{\infty}(0, T]$ and $\left|\partial_{t}^{h} b(t)\right| \leq{ }^{\exists} C_{h}\left|p(t) p^{\prime}(t)\right|^{h}$ for $h=1,2, \ldots, 0<t \leq T$.

The function $p(t)$ implies the speed of degeneracy while the function $b(t)$ describes the oscillations. Typical examples are the following:

$a_{1}(t)=\left\{\begin{array}{ll}\exp \left(-t^{-\alpha}\right) \tilde{b}\left(t^{-1}\right) & \text { for } 0<t \leq T, \\ 0 & \text { for } t=0,\end{array} \quad a_{2}(t)= \begin{cases}t^{\gamma} \tilde{b}\left((-\log t)^{\beta}\right) & \text { for } 0<t \leq T, \\ 0 & \text { for } t=0,\end{cases}\right.$

where $\alpha>0, \beta>0, \gamma>1$ and $\tilde{b}(s)$ is a non-constant, uniformly positive, smooth and 1-periodic function on $(0, \infty)$.

In this paper we shall consider

$$
\begin{cases}\partial_{t}^{2} u-a(t)^{2} \partial_{x}^{2} u=0 & \text { in }[0, T] \times \mathbb{R}_{x}, \\ u(0, x)=u_{0}(x), \partial_{t} u(0, x)=u_{1}(x) & \text { in } \mathbb{R}_{x} .\end{cases}
$$

By $H^{s}\left(\mathbb{R}_{x}\right)$ we denote the Sobolev space equipped with the norm

$$
\|u\|_{s}:=\left(\int_{\mathbb{R}} \sum_{k \leq s}\left|\partial_{x}^{k} u(x)\right|^{2} d x\right)^{1 / 2} .
$$

Further $H^{\infty}\left(\mathbb{R}_{x}\right):=\bigcap_{s \in \mathbb{R}} H^{s}\left(\mathbb{R}_{x}\right)$.

TheOREM 1.1.

a) Assume that $b(t)$ satisfies (1.6). Then for every $u_{0}, u_{1} \in H^{\infty}\left(\mathbb{R}_{x}\right)$, the Cauchy problem (1.7) has a unique solution $u \in C^{2}\left([0, T], H^{\infty}\left(\mathbb{R}_{x}\right)\right)$ represented as follows:

$$
u(t, x)=\sum_{l=0,1} \sum_{m=1,2} \frac{1}{2 \pi} \int_{\mathbb{R}} \int_{\mathbb{R}} e^{i\left[(x-y) \cdot \xi+\varphi_{m}(t, \xi)\right]} a_{l m}(t, \xi) u_{l}(y) d y d \xi,
$$

where $\varphi_{m}(t, \xi)=(-1)^{m} \int_{0}^{t} a(\tau) d \tau \cdot \xi$ for $m=1,2$, while there exist $C_{h \alpha}>0, M>0$ and $0 \leq \rho_{1}<\rho_{2} \leq 1$ such that

(1.8) $\sup _{\substack{0 \leq t \leq T,|\xi| \geq 1}}\left|D_{t}^{h} D_{\xi}^{\alpha} a_{l m}(t, \xi)\right| \leq C_{h \alpha}\langle\xi\rangle^{M+\rho_{1} h-\rho_{2} \alpha} \quad$ for $\alpha \geq 0, \quad h, l=0,1, \quad m=1,2$.

b) The representation is valid for every $u_{0}, u_{1} \in H^{M}\left(\mathbb{R}_{x}\right)$, and

$$
\mathrm{WF}(u(t)) \subset\left\{(x, \xi)=\left(y \pm \int_{0}^{t} a(\tau) d \tau, \eta\right):(y, \eta) \in \mathrm{WF}\left(u_{0}\right) \cup \mathrm{WF}\left(u_{1}\right)\right\} .
$$


c) The problem possesses the finite propagation speed property that for smooth coefficient $a^{2} \in C^{\infty}([0, T])$ together with a) leads to $C^{\infty}$ well-posedness, that is, for every $u_{0}, u_{1} \in C^{\infty}\left(\mathbb{R}_{x}\right)$, the Cauchy problem (1.7) has a unique solution $u \in C^{\infty}\left([0, T] \times \mathbb{R}_{x}\right)$.

REMARK 1.2. In particular when $a(t) \equiv a_{1}(t)$ (resp. $a_{2}(t)$ ), the condition (1.6) corresponds to $\alpha \geq 1 / 2$ (resp. $\beta \leq 2$ ). For $\alpha<1 / 2$ according to [6] the Cauchy problem is not $C^{\infty}$ well-posed. The results of a) and c) are optimal. While, the result b) can be obtained from the general theory of Fourier integral operators.

REMARK 1.3. If $\lambda(t)$ vanishes of infinite order, the Cauchy problem (1.7) has a unique solution $u \in C^{\infty}\left([0, T] \times \mathbb{R}_{x}\right)$ and (1.8) holds for any $h \geq 0$.

2. Notation and classes of symbols. In this paper we often use the cut-off functions $\chi(s)$ and $\psi(s)$ such that

$$
\chi(s)=\left\{\begin{array}{ll}
1 & \text { for } 0 \leq s \leq 1, \\
0 & \text { for } s \geq 2,
\end{array} \quad \chi^{\prime}(s) \leq 0 \quad \text { and } \quad \psi(s)=1-\chi(s) .\right.
$$

We define $\Lambda(t)=\int_{0}^{t} \lambda(\tau) d \tau$ and $\Lambda^{*}(t)=\frac{\Lambda(t)}{p(t)}$. Let $N>0$ and $\langle\xi\rangle=\sqrt{e^{2}+|\xi|^{2}}(\geq e)$.

Definition 2.1. The functions $t_{N}(\xi)$ and $\tilde{t}_{N}(\xi)$ are (unique) roots of $\Lambda^{*}(t)\langle\xi\rangle=$ $2 N \log \langle\xi\rangle$ and $\Lambda^{*}(t)\langle\xi\rangle=4 N \log \langle\xi\rangle$, respectively, i.e., $\Lambda^{*}\left(t_{N}(\xi)\right)\langle\xi\rangle=2 N \log \langle\xi\rangle$ and $\Lambda^{*}\left(\tilde{t}_{N}(\xi)\right)\langle\xi\rangle=4 N \log \langle\xi\rangle$.

DeFinition 2.2. We define the hyperbolic zone

$$
Z_{N}(t, \xi)=\left\{(t, \xi) \in[0, T] \times \mathbb{R}_{\xi}: \Lambda^{*}(t)\langle\xi\rangle \geq 2 N \log \langle\xi\rangle \text { and }|\xi| \geq 1\right\} .
$$

Definition 2.3. Let $m_{1}, m_{2}$ and $m_{3}$ be real numbers. We define the spaces of the symbols

$$
\begin{aligned}
S_{N}\left(m_{1}, m_{2}, m_{3}\right) & =\left\{a(t, \xi) \in C^{\infty}: \sup _{(t, \xi) \in Z_{N}} \frac{\langle\xi\rangle^{|\alpha|-m_{1}}\left|D_{t}^{h} D_{\xi}^{\alpha} a(t, \xi)\right|}{\left.\lambda(t)^{m_{2}}\left|p(t) p^{\prime}(t)\right|^{m_{3}+h} \leq C_{h \alpha}\right\},}\right. \\
S_{N}^{-\infty}\left(m_{1}, m_{2}, m_{3}\right) & =\bigcap_{k=0}^{\infty} S_{N}\left(m_{1}-k, m_{2}-k, m_{3}+k\right) .
\end{aligned}
$$

REMARK 2.4. The following properties are known (see [7]).

(i) $S_{N}\left(m_{1}, m_{2}, m_{3}\right) \supset S_{N}\left(m_{1}-k, m_{2}-k, m_{3}+k\right)$ for $k \geq 0$.

(ii) If $a \in S_{N}\left(m_{1}, m_{2}, m_{3}\right)$, then $D_{\xi}^{\alpha} a \in S_{N}\left(m_{1}-|\alpha|, m_{2}, m_{3}\right)$.

(iii) If $a \in S_{N}\left(m_{1}, m_{2}, m_{3}\right)$ and $b \in S_{N}\left(\tilde{m}_{1}, \tilde{m}_{2}, \tilde{m}_{3}\right)$ (resp. $S_{N}^{-\infty}\left(\tilde{m}_{1}, \tilde{m}_{2}, \tilde{m}_{3}\right)$ ), then $a b \in S_{N}\left(m_{1}+\tilde{m}_{1}, m_{2}+\tilde{m}_{2}, m_{3}+\tilde{m}_{3}\right)\left(\operatorname{resp} . S_{N}^{-\infty}\left(m_{1}+\tilde{m}_{1}, m_{2}+\tilde{m}_{2}, m_{3}+\tilde{m}_{3}\right)\right)$.

Proposition 2.5 ([7]). Suppose that for all $k=1,2, \ldots, a_{k} \in S_{N}\left(m_{1}-k+1, m_{2}-\right.$ $\left.k+1, m_{3}+k-1\right), a_{k}(t, \xi)=0$ for $0 \leq t \leq t_{N}(\xi),|\xi| \geq 1$. Then there exists a symbol $a(t, \xi) \in S_{N}\left(m_{1}, m_{2}, m_{3}\right)$ such that

$$
\operatorname{supp} a \subset Z_{N}(t, \xi) \text { and } a \sim a_{1}+a_{2}+\ldots \bmod S_{N}^{-\infty}\left(m_{1}, m_{2}, m_{3}\right)
$$

in the sense that $a-a_{1}-\ldots-a_{k} \in S_{N}\left(m_{1}-k, m_{2}-k, m_{3}+k\right)$ for all $k=0,1, \ldots$ 
3. Reduction to a first order diagonal system. Without loss of generality we may suppose that $b(t)$ has the form

$$
b(t) \equiv 1+c(t),
$$

where $c(t)$ is a smooth function satisfying

$$
|c(t)| \leq 1 / 2, c \in C^{\infty}(0, T] \quad \text { and } \quad\left|\partial_{t}^{h} c(t)\right| \leq C_{h}\left|p(t) p^{\prime}(t)\right|^{h}\left({ }^{\exists} C_{h}>0\right) .
$$

By Fourier transform the Cauchy problem (1.7) is changed into

$$
\begin{cases}\partial_{t}^{2} v+a(t, \xi)^{2} v=0 & \text { in }[0, T] \times \mathbb{R}_{\xi}, \\ v(0, \xi)=v_{0}(\xi), \quad \partial_{t} v(0, \xi)=v_{1}(\xi) & \text { in } \mathbb{R}_{\xi},\end{cases}
$$

where $a(t, \xi)=\lambda(t)\{1+c(t)\}|\xi|(=a(t)|\xi|)$.

Definition 3.1. The functions $\varepsilon=\varepsilon(\xi)$ and $\tilde{\varepsilon}=\tilde{\varepsilon}(\xi)$ are (unique) roots of the equations $\lambda(t)\langle\xi\rangle=1$ and $\lambda(t)\langle\xi\rangle=2$, respectively, i.e., $\lambda(\varepsilon)\langle\xi\rangle=1$ and $\lambda(\tilde{\varepsilon})\langle\xi\rangle=2$.

Definition 3.2. The functions $\delta=\delta(\xi)$ and $\tilde{\delta}=\tilde{\delta}(\xi)$ are (unique) roots of the equations $\Lambda(t)\langle\xi\rangle=N \log \langle\xi\rangle$ and $\Lambda(t)\langle\xi\rangle=2 N \log \langle\xi\rangle$, respectively, i.e., $\Lambda(\delta)\langle\xi\rangle=N \log \langle\xi\rangle$ and $\Lambda(\tilde{\delta})\langle\xi\rangle=2 N \log \langle\xi\rangle$.

LEMma 3.3. For sufficiently large $N>0$, the following relation holds:

$$
0<\varepsilon<\tilde{\varepsilon}<\delta<\tilde{\delta} \leq t_{N}(\xi)<\tilde{t}_{N}(\xi) \text { for } \xi \in \mathbb{R}_{\xi}
$$

Now we approximate $a(t, \xi)$ with two functions defined by the following formulas.

$$
\begin{aligned}
a^{*}(t, \xi) & =\chi(\lambda(t)\langle\xi\rangle)+\psi(\lambda(t)\langle\xi\rangle) \lambda(t)\left\{1+\psi\left(\frac{\Lambda(t)\langle\xi\rangle}{N \log \langle\xi\rangle}\right) c(t)\right\}\langle\xi\rangle, \\
a^{* *}(t, \xi) & =\frac{\lambda(\tilde{\delta})\langle\xi\rangle}{2} \chi\left(\frac{\Lambda(t)\langle\xi\rangle}{N \log \langle\xi\rangle}\right)+\psi\left(\frac{\Lambda(t)\langle\xi\rangle}{N \log \langle\xi\rangle}\right) \lambda(t)\left\{1+\psi\left(\frac{\Lambda(t)\langle\xi\rangle}{N \log \langle\xi\rangle}\right) c(t)\right\}\langle\xi\rangle .
\end{aligned}
$$

Noting that $|c(s)| \leq 1 / 2$ and considering the supports of $\chi$ and $\psi$, we get

Lemma 3.4. For any $t \in[0, T]$ and $\xi \in \mathbb{R}_{\xi}, a^{*}(t, \xi)$ and $a^{* *}(t, \xi)$ are positive, more precisely

$$
\begin{aligned}
& a^{*}(t, \xi) \geq \max \left\{\frac{\lambda(t)\langle\xi\rangle}{2}, \frac{1}{2}\right\}, \quad a^{* *}(t, \xi) \geq \max \left\{\frac{\lambda(t)\langle\xi\rangle}{2}, \frac{\lambda(\delta)\langle\xi\rangle}{2}\right\} \\
& \left|D_{t}^{h} D_{\xi}^{\alpha} a^{*}(t, \xi)\right| \leq C_{h \alpha}\langle\xi\rangle^{-|\alpha|}\left(\max \left\{\left|p^{\prime}(\varepsilon)\right|,\left|p^{\prime}(\delta)\right| p(\delta)\right\}\right)^{h} a^{*}(t, \xi) \\
& \left|D_{t}^{h} D_{\xi}^{\alpha} a^{* *}(t, \xi)\right| \leq C_{h \alpha}\langle\xi\rangle^{-|\alpha|}\left(\left|p^{\prime}(\delta)\right| p(\delta)\right)^{h} a^{* *}(t, \xi) .
\end{aligned}
$$

Putting $W=\left(\begin{array}{c}a^{* *}(t, \xi)^{1 / 2} v \\ \partial_{t}\left\{a^{* *}(t, \xi)^{1 / 2} v\right\}\end{array}\right)$ and $W_{0}:=\left.W\right|_{t=0}=\left\{\frac{\lambda(\tilde{\delta})\langle\xi\rangle}{2}\right\}^{1 / 2}\left(\begin{array}{c}v_{0} \\ v_{1}\end{array}\right)$ and multiplying both sides of $(3.1)$ by $a^{* *}(t, \xi)^{1 / 2}$, we find that the Cauchy problem (3.1) is equivalent to the one for the system,

$$
\left\{\begin{array}{l}
\partial_{t} W=\left(\begin{array}{cc}
0 & 1 \\
-a(t, \xi)^{2}-r(t, \xi) & \frac{\partial_{t} a^{* *}(t, \xi)}{a^{* *}(t, \xi)}
\end{array}\right) W, \\
W(0)=W_{0},
\end{array}\right.
$$

where $r(t, \xi)=\frac{3}{4}\left(\frac{\partial_{t} a^{* *}(t, \xi)}{a^{* *}(t, \xi)}\right)^{2}-\frac{1}{2} \frac{\partial_{t}^{2} a^{* *}(t, \xi)}{a^{* *}(t, \xi)}$. 
Further we make one step of the diagonalization by putting

$$
W^{*}=\left(\begin{array}{cc}
1 & 1 \\
-i a^{*}(t, \xi) & i a^{*}(t, \xi)
\end{array}\right)^{-1} W \quad \text { and } \quad W_{0}^{*}:=\left.W^{*}\right|_{t=0}=\frac{1}{2}\left(\begin{array}{cc}
1 & i \\
1 & -i
\end{array}\right) W_{0},
$$

and multiplying both sides of the system by $\left(\begin{array}{cc}1 & 1 \\ -i a^{*}(t, \xi) & i a^{*}(t, \xi)\end{array}\right)^{-1}$, where $a^{*}(t, \xi)^{-1}$ belongs to $S_{N}(-1,-1,0)$. Then we obtain

$$
\left\{\begin{array}{l}
\partial_{t} W^{*}=D(t, \xi) W^{*}+B(t, \xi) W^{*}, \\
W^{*}(0)=W_{0}^{*}
\end{array}\right.
$$

where $D=i a(t, \xi)\left(\begin{array}{cc}-1 & 0 \\ 0 & 1\end{array}\right)$,

$$
\begin{aligned}
B= & \frac{1}{2}\left\{\frac{\partial_{t} a^{* *}(t, \xi)}{a^{* *}(t, \xi)}-\frac{\partial_{t} a^{*}(t, \xi)}{a^{*}(t, \xi)}\right\}\left(\begin{array}{cc}
1 & -1 \\
-1 & 1
\end{array}\right) \\
& +i\left\{a^{*}(t, \xi)-a(t, \xi)\right\}\left(\begin{array}{cc}
-1 & 0 \\
0 & 1
\end{array}\right)+\frac{a(t, \xi)^{2}-a^{*}(t, \xi)^{2}+r(t, \xi)}{2 i a^{*}(t, \xi)}\left(\begin{array}{cc}
1 & 1 \\
-1 & -1
\end{array}\right) .
\end{aligned}
$$

Definition 3.5. We define recursively the sequence $\left\{B^{(k)}(t, \xi)\right\}_{k \geq 1}$ as

$$
\left\{\begin{array}{l}
B^{(1)}(t, \xi)=B(t, \xi)-\chi\left(\frac{\Lambda^{*}(t)\langle\xi\rangle}{2 N \log \langle\xi\rangle}\right) B(t, \xi) \\
B^{(k)}(t, \xi)=\tilde{B}^{(k)}(t, \xi)-\chi\left(\frac{\Lambda^{*}(t)\langle\xi\rangle}{2 N \log \langle\xi\rangle}\right) B(t, \xi) \quad \text { for } k=2,3, \ldots
\end{array}\right.
$$

where

$$
\begin{aligned}
\tilde{B}^{(k)}(t, \xi)=\left(I+\sum_{j=1}^{k-1} H^{(j)}(t, \xi)\right)\left(\partial_{t}-D(t, \xi)-\sum_{j=1}^{k-1} F^{(j)}(t, \xi)\right) & \\
& -\left(\partial_{t}-D(t, \xi)-B(t, \xi)\right)\left(I+\sum_{j=1}^{k-1} H^{(j)}(t, \xi)\right), \\
F^{(k)}(t, \xi)=\operatorname{diag} B^{(k)}(t, \xi), & H^{(k)}(t, \xi)=\frac{1}{2 i a(t, \xi)}\left(\begin{array}{cc}
0 & B_{12}^{(k)}(t, \xi) \\
-B_{21}^{(k)}(t, \xi) & 0
\end{array}\right) .
\end{aligned}
$$

When $0 \leq t \leq t_{N}(\xi)$, we see that $B^{(1)}(t, \xi)=0$ and recursively $B^{(k)}(t, \xi)=0$ for $k=1,2, \ldots$. Thus, we obtain $H^{(k)}(t, \xi)=0$ for $k=1,2, \ldots$ Moreover we can derive for $k=2,3, \ldots$

$$
\begin{aligned}
B^{(k)}=\sum_{j=1}^{k-1}\left[D, H^{(j)}\right]-\sum_{j=1}^{k-1} \partial_{t} H^{(j)}-\left(I+\sum_{j=1}^{k-1} H^{(j)}\right) & \sum_{j=1}^{k-1} F^{(j)}+B\left(I+\sum_{j=1}^{k-1} H^{(j)}\right)-\chi B \\
=\left\{B^{(k-1)}+\left[D, H^{(k-1)}\right]-F^{(k-1)}\right\} & +B H^{(k-1)}-\partial_{t} H^{(k-1)} \\
& -\sum_{j=1}^{k-1} H^{(k-1)} F^{(j)}-\sum_{j=1}^{k-2} H^{(j)} F^{(k-1)} .
\end{aligned}
$$

Noting that $B^{(k-1)}+\left[D, H^{(k-1)}\right]-F^{(k-1)} \equiv 0$, we also obtain recursively the following 
Lemma 3.6. For sufficiently large $N>0$,

$B^{(k)} \in S_{N}(-k,-k, k+1), \quad H^{(k)} \in S_{N}(-k-1,-k-1, k+1) \quad$ for $k=1,2, \ldots$,

$F^{(k)} \in \begin{cases}S_{N}(-1,-1,2) & \text { for } k=1, \\ S_{N}(-k-1,-k-1, k+2) & \text { for } k=2,3, \ldots\end{cases}$

By Proposition 2.5 there exists $H(t, \xi)=\left(\begin{array}{cc}0 & H_{12} \\ H_{21} & 0\end{array}\right) \in S_{N}(-2,-2,2)$ such that supp $H \subset Z_{N}(t, \xi)$ and $H \sim H^{(1)}+H^{(2)}+\ldots \bmod S_{N}^{-\infty}(-2,-2,2)$, and for sufficiently large $N>0$, there exists $H^{*}(t, \xi) \in S_{N}(-2,-2,2)$ defined by

$$
H^{*}(t, \xi) \equiv \frac{1}{1-H_{12} H_{21}}\left(\begin{array}{cc}
H_{12} H_{21} & -H_{12} \\
-H_{21} & H_{12} H_{21}
\end{array}\right)=(I+H(t, \xi))^{-1}-I .
$$

Finally, putting

$$
W^{* *}=\left(I+H^{*}(t, \xi)\right) W^{*} \quad \text { and } \quad W_{0}^{* *}:=\left.W^{* *}\right|_{t=0}=\left(I+H^{*}(0, \xi)\right) W_{0}^{*}=W_{0}^{*},
$$

and multiplying both sides of $(3.2)$ by $\left(I+H^{*}(t, \xi)\right)$, we obtain

$$
\left\{\begin{aligned}
\partial_{t} W^{* *}=D(t, \xi) W^{* *} & +\operatorname{diag}\left\{B^{*}(t, \xi)\right\} W^{* *}+\chi\left(\frac{\Lambda^{*}(t)\langle\xi\rangle}{2 N \log \langle\xi\rangle}\right) B(t, \xi) W^{* *} \\
& +\chi\left(\frac{\Lambda^{*}(t)\langle\xi\rangle}{2 N \log \langle\xi\rangle}\right) B^{* *}(t, \xi) W^{* *}+R(t, \xi) W^{* *}, \\
W^{* *}(0)=W_{0}^{* *}, &
\end{aligned}\right.
$$

where $R(t, \xi) \subset S_{N}^{-\infty}(-1,-1,2), B^{*}(t, \xi)=B^{(1)}+B H \in S_{N}(-1,-1,2)$ and $B^{* *}(t, \xi)=$ $H^{*} B \in S_{N}(-3,-3,4)$.

4. Some estimates. In this section we show some estimates which will be used to construct the fundamental solution of (3.3). For simplicity we define $\chi^{*}(t)=\chi(\lambda(t)\langle\xi\rangle)$, $\chi^{* *}(t)=\chi\left(\frac{\Lambda(t)\langle\xi\rangle}{N \log \langle\xi\rangle}\right)$ and $\psi^{*}(t)=1-\chi^{*}(t), \psi^{* *}(t)=1-\chi^{* *}(t)$.

Noting the support of $\chi\left(\frac{\Lambda^{*}(t)\langle\xi\rangle}{2 N \log \langle\xi\rangle}\right)$, we obtain

$$
\begin{aligned}
\int_{0}^{T} \chi\left(\frac{\Lambda^{*}(t)\langle\xi\rangle}{2 N \log \langle\xi\rangle}\right) B(t, \xi) d t & \leq \frac{1}{2} \int_{0}^{\tilde{t}_{N}(\xi)}\left|\frac{\partial_{t} a^{* *}}{a^{* *}}-\frac{\partial_{t} a^{*}}{a^{*}}\right| d t+\int_{0}^{\tilde{t}_{N}(\xi)}\left|a^{*}-a\right| d t \\
& +\frac{1}{2} \int_{0}^{\tilde{t}_{N}(\xi)} \frac{\left|a^{2}-a^{* 2}\right|}{a^{*}} d t+\frac{1}{2} \int_{0}^{\tilde{t}_{N}(\xi)} \frac{|r|}{a^{*}} d t \\
& \equiv I_{1}+I_{2}+I_{3}+I_{4} .
\end{aligned}
$$

Since $\partial_{t} a^{*}(t, \xi)=0$ for $0 \leq t \leq \varepsilon, \partial_{t} a^{* *}(t, \xi)=0$ for $0 \leq t \leq \delta$ and $a^{*}(t, \xi)=a^{* *}(t, \xi)$ for $\tilde{\delta} \leq t\left(\leq \tilde{t}_{N}(\xi)\right)$, we deduce that

$$
I_{1}=\frac{1}{2} \int_{\varepsilon}^{\tilde{\delta}}\left|\frac{\partial_{t} a^{* *}}{a^{* *}}-\frac{\partial_{t} a^{*}}{a^{*}}\right| d t \leq \frac{1}{2} \int_{\varepsilon}^{\tilde{\delta}}\left|\frac{\partial_{t} a^{*}}{a^{*}}\right| d t+\frac{1}{2} \int_{\delta}^{\tilde{\delta}}\left|\frac{\partial_{t} a^{* *}}{a^{* *}}\right| d t \equiv I_{1}^{*}+I_{1}^{* *} .
$$

Noting the supports of $\chi^{* \prime}, \psi^{* \prime}$ and $\psi^{* * \prime}$, by (1.6) and Lemma 3.4 we obtain

$$
I_{1}^{*} \leq C \int_{\varepsilon}^{\tilde{\delta}}\left\{\frac{\left|\chi^{* \prime}\right|}{a^{*}}+\frac{\left|\psi^{* \prime}\right| \lambda\langle\xi\rangle}{a^{*}}+\frac{\psi^{*}\left|\lambda^{\prime}\right|\langle\xi\rangle}{a^{*}}+\frac{\left|\psi^{* * \prime}\right| \lambda\langle\xi\rangle}{a^{*}}+\frac{\psi^{* *} \lambda\left|c^{\prime}\right|\langle\xi\rangle}{a^{*}}\right\} d t
$$




$$
\begin{aligned}
& \leq C\left\{\int_{\varepsilon}^{\tilde{\varepsilon}}\left|\chi^{*^{\prime}}\right| d t+\int_{\varepsilon}^{\tilde{\varepsilon}} \lambda^{\prime}\langle\xi\rangle d t+\int_{\varepsilon}^{\tilde{\delta}} \frac{\lambda^{\prime}}{\lambda} d t+\int_{\delta}^{\tilde{\delta}} \frac{\lambda\langle\xi\rangle}{N \log \langle\xi\rangle} d t+\int_{\delta}^{\tilde{\delta}}\left|p p^{\prime}\right| d t\right\} \\
& \leq C\left\{1+1+\int_{\varepsilon}^{\tilde{\delta}}\left|p^{\prime}\right| d t+1+p(\delta) \int_{\delta}^{\tilde{\delta}}\left|p^{\prime}\right| d t\right\} \leq C p(\varepsilon) .
\end{aligned}
$$

Similarly we also obtain $I_{1}^{* *} \leq C p(\varepsilon)$. Thus we get $I_{1} \leq I_{1}^{*}+I_{1}^{* *} \leq C p(\varepsilon)$. Since $a(t, \xi)=$ $a^{*}(t, \xi)$ for $\tilde{\delta} \leq t\left(\leq \tilde{t}_{N}(\xi)\right)$, we get $I_{2}=\int_{0}^{\tilde{\delta}}\left|a^{*}-a\right| d t \leq C \Lambda(\tilde{\delta})\langle\xi\rangle \leq C_{N} \log \langle\xi\rangle$. Similarly, by Lemma 3.4 we get $I_{3} \leq C_{N} \log \langle\xi\rangle$. Since $\partial_{t} a^{* *}(t, \xi)=0, \partial_{t}^{2} a^{* *}(t, \xi)=0$ for $0 \leq t \leq \delta$, we deduce that

$$
I_{4} \leq C \int_{\delta}^{\tilde{t}_{N}(\xi)} \frac{1}{a^{*}}\left|\frac{\partial_{t} a^{* *}}{a^{* *}}\right|^{2} d t+C \int_{\delta}^{\tilde{t}_{N}(\xi)} \frac{1}{a^{*}}\left|\frac{\partial_{t}^{2} a^{* *}}{a^{* *}}\right| d t \equiv I_{4}^{*}+I_{4}^{* *} .
$$

The last estimate corresponds to the estimate in the oscillation's subzone of [5]. Noting the supports of $\chi^{* * \prime}$ and $\psi^{* * \prime}$, by (1.6) and Lemma 3.4 we obtain

$$
\begin{aligned}
I_{4}^{*} & \leq C \int_{\delta}^{\tilde{t}_{N}}\left\{\frac{\left|\lambda(\tilde{\delta})\langle\xi\rangle \chi^{* * \prime}\right|^{2}}{a^{*} a^{* * 2}}+\frac{\left|\psi^{* * \prime}\right|^{2} \lambda^{2}\langle\xi\rangle^{2}}{a^{*} a^{* * 2}}+\frac{\psi^{* *}\left|\lambda^{\prime}\right|^{2}\langle\xi\rangle^{2}}{a^{*} a^{* * 2}}+\frac{\psi^{* *} \lambda^{2}\left|c^{\prime}\right|^{2}\langle\xi\rangle^{2}}{a^{*} a^{* * 2}}\right\} d t \\
& \leq C\left\{\int_{\delta}^{\tilde{\delta}} \frac{\lambda\langle\xi\rangle}{(N \log \langle\xi\rangle)^{2}} d t+\int_{\delta}^{\tilde{t}_{N}}\left(\frac{\lambda^{\prime}}{\lambda}\right)^{2} \frac{1}{\lambda\langle\xi\rangle} d t+\int_{\delta}^{\tilde{t}_{N}} \frac{\left|p p^{\prime}\right|^{2}}{\lambda\langle\xi\rangle} d t\right\} \leq C \int_{\delta}^{\tilde{t}_{N}} \frac{p^{2}\left|p^{\prime}\right|^{2}}{\lambda\langle\xi\rangle} d t .
\end{aligned}
$$

Similarly we also obtain $I_{4}^{* *} \leq C \int_{\delta}^{\tilde{t}_{N}} \frac{p^{2}\left|p^{\prime}\right|^{2}}{\lambda\langle\xi\rangle} d t$. Thus $I_{4} \leq I_{4}^{*}+I_{4}^{* *} \leq C \int_{\delta}^{\tilde{t}_{N}(\xi)} \frac{p^{2}\left|p^{\prime}\right|^{2}}{\lambda\langle\xi\rangle} d t \leq$ $C \frac{p(\delta)^{2}\left|p^{\prime}(\delta)\right|}{\langle\xi\rangle} \int_{\delta}^{\tilde{t}_{N}}\left(-p^{\prime}(t)\right) e^{p(t)} d t \leq C p(\delta)^{2}\left|p^{\prime}(\delta)\right| e^{p(\delta)}\langle\xi\rangle^{-1}$.

Combining $I_{1}-I_{4}$ and noting that $\left|p^{\prime}(\delta)\right| e^{p(\delta)} \leq \frac{\langle\xi\rangle}{N \log \langle\xi\rangle}$, we have for $\xi \in \mathbb{R}_{\xi}$

$$
\begin{array}{r}
\int_{0}^{T} \chi\left(\frac{\Lambda^{*}(t)\langle\xi\rangle}{2 N \log \langle\xi\rangle}\right) B d t \leq C p(\varepsilon)+C_{N} \log \langle\xi\rangle+C p(\delta)^{2}\left|p^{\prime}(\delta)\right| e^{p(\delta)}\langle\xi\rangle^{-1} \\
\leq C_{N} \log \langle\xi\rangle .
\end{array}
$$

Noting the supports of $\chi\left(\frac{\Lambda^{*}(t)\langle\xi\rangle}{2 N \log \langle\xi\rangle}\right)$ and observing that $B^{* *}(t, \xi) \in S_{N}(-3,-3,4)$, we obtain for $\xi \in \mathbb{R}_{\xi}$

$$
\int_{0}^{T} \chi\left(\frac{\Lambda^{*}(t)\langle\xi\rangle}{2 N \log \langle\xi\rangle}\right) B^{* *} d t \leq C \int_{t_{N}}^{\tilde{t}_{N}} \frac{p(t)^{4}\left|p^{\prime}(t)\right|^{4}}{\langle\xi\rangle^{3} \lambda(t)^{3}} d t=C \int_{t_{N}}^{\tilde{t}_{N}} \frac{p(t)\left|p^{\prime}(t)\right|}{\left(\Lambda^{*}(t)\langle\xi\rangle\right)^{3}} d t \leq C
$$

Since $R(t, \xi) \in S_{N}^{-\infty}(-1,-1,2) \in S_{N}(-3,-3,4)$, similarly we have for $\xi \in \mathbb{R}_{\xi}$

$$
\int_{0}^{T} R d t \leq C \int_{t_{N}}^{T} \frac{p(t)^{k+2}\left|p^{\prime}(t)\right|^{k+2}}{\langle\xi\rangle^{k+1} \lambda(t)^{k+1}} d t=C \int_{t_{N}}^{T} \frac{p(t)\left|p^{\prime}(t)\right|}{\left(\Lambda^{*}(t)\langle\xi\rangle\right)^{k+1}} d t
$$

5. The representation formula. We define the diagonal matrix function

$$
E(t, \xi)=\exp \left\{\int_{0}^{t} D(\tau, \xi) d \tau\right\}=\left(\begin{array}{cc}
\exp \left\{-i \int_{0}^{t} a(\tau, \xi) d \tau\right\} & 0 \\
0 & \exp \left\{i \int_{0}^{t} a(\tau, \xi) d \tau\right\}
\end{array}\right) .
$$

For every $\Psi(\xi)$ the vector function $V(t, \xi)=E(t, \xi) \Psi(\xi)$ is a solution of the Cauchy problem

$$
\left\{\begin{array}{l}
\partial_{t} V=D(t, \xi) V \quad \text { in }[0, T] \times \mathbb{R}_{\xi} \\
V(0, \xi)=\Psi(\xi)
\end{array}\right.
$$


Now we put

$$
G(t, \xi) \equiv E^{-1}\left\{\operatorname{diag}\left\{B^{*}\right\}+\chi\left(\frac{\Lambda^{*}(t)\langle\xi\rangle}{2 N \log \langle\xi\rangle}\right) B+\chi\left(\frac{\Lambda^{*}(t)\langle\xi\rangle}{2 N \log \langle\xi\rangle}\right) B^{* *}+R\right\} E .
$$

Then the matrix function

$$
K(t, \xi)=\sum_{j=1}^{\infty} \int_{0}^{t} G\left(t_{1}, \xi\right) d t_{1} \int_{0}^{t_{1}} G\left(t_{2}, \xi\right) d t_{2} \cdots \int_{0}^{t_{j-1}} G\left(t_{j}, \xi\right) d t_{j}
$$

is the solution of the Cauchy problem

$$
\left\{\begin{array}{l}
\partial_{t} K(t, \xi)=G(t, \xi) K(t, \xi)+G(t, \xi) \quad \text { in }[0, T] \times \mathbb{R}_{\xi} \\
K(0, \xi)=0
\end{array}\right.
$$

Moreover by (4.1), (4.2) and (4.3) we get the following result.

Proposition 5.1. There exist $M_{N}>0$ and a strictly increasing function $\phi(s)$ satisfying $\lim _{s \rightarrow+\infty} \phi(s) / s=0$ such that

$$
\sup _{0 \leq t \leq T,|\xi| \geq 1}\left|D_{t}^{h} D_{\xi}^{\alpha} K\right| \leq C_{N h \alpha}\langle\xi\rangle^{M_{N}} \phi(\langle\xi\rangle)^{h}\left\{\frac{(\log \langle\xi\rangle)^{2}}{\langle\xi\rangle}\right\}^{\alpha} \quad \text { for } h=0,1, \quad \alpha \geq 0 .
$$

REMARK 5.2. If we take $\phi(s)=\lambda\left(\Lambda^{*-1}\left(\frac{4 N \log s}{s}\right)\right) s$ which is a strictly increasing function and satisfies $\lim _{s \rightarrow+\infty} \phi(s) / s=0$, then (5.3) holds. In case that $a(t) \equiv a_{1}(t)$ $\left(\right.$ resp. $\left.a_{2}(t)\right)($ see Section 1$)$, we can set $\phi(s)=C(\log s)^{3+1 / \alpha}\left(\operatorname{resp} . C(\log s)^{2} s^{1 / \gamma}\right)$.

By (5.1) and (5.2) we have

$$
\begin{aligned}
\partial_{t}\{E(t, \xi)(I+K(t, \xi)) \Psi(\xi)\} & =\partial_{t}(E \Psi)+\left(\partial_{t} E\right) K \Psi+E\left(\partial_{t} K\right) \Psi \\
& =D(E \Psi)+D K \Psi+E(G K+G) \Psi \\
& =D\{E(I+K) \Psi\}+E G E^{-1}\{E(I+K) \Psi\} .
\end{aligned}
$$

This means that the matrix function $E(t, \xi)(I+K(t, \xi))$ is the fundamental solution of the Cauchy problem (3.3). Thus we have the following statement.

Theorem 5.3. Assume that $b(t)$ satisfies (1.6). Then the solution $v(t, \xi)$ to the Cauchy problem (3.1) can be represented as

$$
v(t, \xi)=\left\{\frac{\lambda(\tilde{\delta})\langle\xi\rangle}{8 a^{* *}}\right\}^{1 / 2}\left(H_{21}(t, \xi)+1, H_{12}(t, \xi)+1\right) E(t, \xi)(I+K(t, \xi))\left(\begin{array}{l}
v_{0}+i v_{1} \\
v_{0}-i v_{1}
\end{array}\right),
$$

and

$\sup _{0 \leq t \leq T,|\xi| \geq 1}\left|D_{t}^{h} D_{\xi}^{\alpha} v(t, \xi)\right| \leq C\langle\xi\rangle^{M_{N}} \phi(\langle\xi\rangle)^{h}\left\{\frac{(\log \langle\xi\rangle)^{2}}{\langle\xi\rangle}\right\}^{\alpha}\left|v_{l}(\xi)\right| \quad$ for $\quad \alpha \geq 0, h, l=0,1$.

Proof. From the definitions we obtain $W_{0}^{* *}=\frac{\lambda(\tilde{\delta})^{1 / 2}\langle\xi\rangle^{1 / 2}}{2 \sqrt{2}}\left(\begin{array}{cc}1 & i \\ 1 & -i\end{array}\right)\left(\begin{array}{l}v_{0} \\ v_{1}\end{array}\right)$,

$$
W^{* *}=\left(I+H^{*}\right)\left(\begin{array}{cc}
1 & 1 \\
-i a^{*} & i a^{*}
\end{array}\right)^{-1}\left(\begin{array}{cc}
a^{* * 1 / 2} & 0 \\
\frac{\partial_{t} a^{* * 1 / 2}}{2 a^{* * 1 / 2}} & a^{* * 1 / 2}
\end{array}\right)\left(\begin{array}{c}
v \\
\partial_{t} v
\end{array}\right) \text {. }
$$

Therefore we get

$$
\left(\begin{array}{c}
v(t, \xi) \\
\partial_{t} v(t, \xi)
\end{array}\right)=\left(\begin{array}{cc}
a^{* *}(t, \xi)^{1 / 2} & 0 \\
\frac{\partial_{t} a^{* *}(t, \xi)^{1 / 2}}{2 a^{* *}(t, \xi)^{1 / 2}} & a^{* *}(t, \xi)^{1 / 2}
\end{array}\right)^{-1}\left(\begin{array}{cc}
1 & 1 \\
-i a^{*}(t, \xi) & i a^{*}(t, \xi)
\end{array}\right)(I+H(t, \xi))
$$




$$
\times E(t, \xi)(I+K(t, \xi)) \frac{\lambda(\tilde{\delta})^{1 / 2}\langle\xi\rangle^{1 / 2}}{2 \sqrt{2}}\left(\begin{array}{cc}
1 & i \\
1 & -i
\end{array}\right)\left(\begin{array}{l}
v_{0}(\xi) \\
v_{1}(\xi)
\end{array}\right) .
$$

Hence we obtain the representation formula of $v(t, \xi)$. Moreover by Lemma 3.4, Proposition 5.1 we get for $0 \leq t \leq T$

$$
\begin{aligned}
& |v(t, \xi)| \leq C\left|\frac{\lambda(\tilde{\delta})\langle\xi\rangle}{2 a^{* *}(t, \xi)}\right|^{1 / 2}\left(\max \left\{\left|H_{12}\right|,\left|H_{12}\right|\right\}+1\right)|E|(1+|K|)\left(\left|\hat{u}_{0}\right|+\left|\hat{u}_{1}\right|\right) \\
& \quad \leq C\left|\frac{\lambda(\tilde{\delta})\langle\xi\rangle}{2 \frac{\lambda(\delta)\langle\xi\rangle}{2}}\right|^{1 / 2} \cdot(1+1) \cdot 1 \cdot\left(1+C\langle\xi\rangle^{M_{N}}\right)\left(\left|\hat{u}_{0}\right|+\left|\hat{u}_{1}\right|\right) \leq C\langle\xi\rangle^{M_{N}}\left(\left|\hat{u}_{0}\right|+\left|\hat{u}_{1}\right|\right) .
\end{aligned}
$$

Similarly, Lemma 3.4 and Proposition 5.1 give the estimate of derivatives.

Finally, by Theorem 5.3 we can conclude the proof of Theorem 1.1 with

$$
\begin{aligned}
& a_{l 1}=\left\{\begin{array}{ll}
i^{l}\left\{\frac{\lambda(\tilde{\delta})\langle\xi\rangle}{8 a^{* *}}\right\}^{1 / 2}\left(H_{21}(t, \xi)+1\right)(I+K(t, \xi)) & \text { if } \xi \geq 0, \\
(-i)^{l}\left\{\frac{\lambda(\tilde{\delta})\langle\xi\rangle}{8 a^{* *}}\right\}^{1 / 2}\left(H_{12}(t, \xi)+1\right)(I+K(t, \xi)) & \text { if } \xi<0,
\end{array} \text { for } \quad l=1,2,\right. \\
& a_{l 2}=\left\{\begin{array}{ll}
(-i)^{l}\left\{\frac{\lambda(\tilde{\delta})\langle\xi\rangle}{8 a^{* *}}\right\}^{1 / 2}\left(H_{12}(t, \xi)+1\right)(I+K(t, \xi)) & \text { if } \xi \geq 0, \\
i^{l}\left\{\frac{\lambda(\tilde{\delta})\langle\xi\rangle}{8 a^{* *}}\right\}^{1 / 2}\left(H_{21}(t, \xi)+1\right)(I+K(t, \xi)) & \text { if } \xi<0,
\end{array} \text { for } \quad l=1,2 .\right.
\end{aligned}
$$

Acknowledgments. This paper was written during the work of the second author as a Foreign Professor at the University of Tsukuba. He is very grateful to all the members of Institute of Mathematics of the University of Tsukuba for the helpful and warm hospitality. Special thanks to Professor Michael Reissig for very useful discussions.

\section{References}

[1] F. Colombini and S. Spagnolo, An example of a weakly hyperbolic Cauchy problem not well posed in $C^{\infty}$, Acta Math. 148 (1982), 243-253.

[2] F. Colombini and S. Spagnolo, Hyperbolic equations with coefficients rapidly oscillating in time: a result of nonstability, J. Differential Equations 52 (1984), 24-38.

[3] K. Kajitani, The well posed Cauchy problem for hyperbolic operators, Exposé au Séminaire de Vaillant du 8 février 1989, 1-16.

[4] M. Reissig and K. Yagdjian, One application of Floquet's theory to $L_{p}-L_{q}$ estimates for hyperbolic equations with very fast oscillations, Math. Methods Appl. Sci. 22 (1999), 937-951.

[5] M. ReIssig and K. YAGDJiAn, $L_{p}-L_{q}$ decay estimates for hyperbolic equations with oscillations in coefficients, Chinese Ann. Math. Ser. B 21 (2000), 153-164.

[6] S. TARAma, On the second order hyperbolic equations degenerating in the infinite order. Example, Math. Japon. 42 (1995), 523-533.

[7] K. Yagdjian, The Cauchy Problem for Hyperbolic Operators. Multiple Characteristics. Micro-local Approach, Math. Top. 12, Akademie Verlag, Berlin, 1997.

[8] K. Yagdjian, Representation theorem for the solutions of equations with the turning point of infinite order, Ann. Mat. Pura Appl. (4) 173 (1997), 13-30. 